\title{
TRATAMENTO DO CARCINOMA DO CANAL ANAL COM RADIOTERAPIA E QUIMIOTERAPIA CONCOMITANTES. RESULTADOS PRELIMINARES DO HOSPITAL DO CÂNCER A. C. CAMARGO
}

\author{
TREATMENT OF ANAL CANAL CARCINOMA WITH CONCOMITANT \\ RADIOTHERAPY AND CHEMOTHERAPY. PRELIMINARY RESULTS FROM \\ HOSPITAL DO CÂNCER A. C. CAMARGO
}

\author{
Carlos Genésio Bezerra Lima Júnior ${ }^{1}$ \\ Robson Ferrigno ${ }^{2}$ \\ João Victor Salvajoli ${ }^{3}$ \\ Waldec Jorge David Filho ${ }^{4}$ \\ Benedito Mauro Rossi, TCBC-SP 5 \\ Ademar Lopes, TCBC-SP ${ }^{6}$
}

\begin{abstract}
RESUMO: Objetivo: Reportar os resultados preliminares do tratamento do carcinoma do canal anal com radioterapia e quimioterapia concomitantes. Métodos: De janeiro de 1992 a maio de 1998, foram tratados 24 pacientes com diagnóstico histológico de carcinoma do canal anal, sendo 18 pacientes do sexo feminino e seis do sexo masculino (3:1). A idade dos mesmos variou de 35 a 74 anos e a média foi de 59 anos. A distribuição do número de pacientes por estádio clínico foi: I - 1, II - 13, III - 9 e IV - 1. A radioterapia foi realizada com dose de 45 Gy na pelve no Acelerador Linear de $4 \mathrm{MV}$, seguida de complementação de dose no canal anal até 55 Gy através de campo direto no cobalto. A quimioterapia foi realizada com 5-FU $\left(1.000 \mathrm{mg} / \mathrm{m}^{2}\right)$ em infusão contínua e mitomicina C $\left(10 \mathrm{mg} / \mathrm{m}^{2}\right)$ em bólus durante os cinco primeiros e os cinco últimos dias da radioterapia. Resultados: $\mathrm{O}$ seguimento médio foi de 34 meses. Resposta completa ao tratamento foi obtida em $23(95,8 \%)$ pacientes. Quatorze $(58,3 \%)$ estão vivos sem doença, três $(12 \%)$ vivos com doença, cinco $(20,8 \%)$ mortos pelo câncer e um $(4,2 \%)$ morreu sem câncer. Recidivas locais ocorreram em cinco (20,8\%) pacientes e metástase a distância em quatro $(16,6 \%)$. A função esfincteriana foi preservada em 18 (75\%) pacientes. Complicações agudas e crônicas foram observadas em 19 (79,2\%) e em nove (37,5\%) pacientes respectivamente. Conclusões: O tratamento foi efetivo em termos de controle local e preservação esfincteriana, porém com toxicidade aguda e tardia elevadas. Diminuição da dose de radioterapia em toda pelve representa uma estratégia razoável para diminuir os efeitos colaterais agudos e crônicos. Um maior número de pacientes e seguimento mais longo trarão mais informações sobre esta abordagem terapêutica.
\end{abstract}

Descritores: Carcinoma; Canal anal; Radioterapia; Quimioterapia.

\section{INTRODUÇÃO}

O carcinoma do canal anal representa uma doença cuja abordagem terapêutica evoluiu notavelmente nos últimos 30 anos em função dos resultados de preservação da função esfincteriana. Constitui uma neoplasia pouco freqüente do trato gastrointestinal inferior, respondendo por menos de 5\% dos tumores deste sistema, predominando no sexo feminino na proporção de 2:1 a 5:1 (feminino/ masculino) na dependência da série considerada ${ }^{1,2}$. A ida-

1. Residente do Departamento de Radioterapia

2. Titular do Departamento de Radioterapia

3. Diretor do Departamento de Radioterapia

4. Titular do Departamento de Oncologia Clínica

5. Titular do Departamento de Cirurgia Pélvica.

6. Diretor do Departamento de Cirurgia Pélvica.

Recebido em 16/9/1999

Aceito para publicação em 19/9/2000

Trabalho realizado no Centro de Tratamento e Pesquisa do Hospital do Câncer A. C. Camargo - São Paulo-SP. 
de principal de acometimento situa-se ao redor da sexta e da sétima décadas de vida ${ }^{3}$.

A literatura não é precisa no que concerne à definição de canal anal e margem anal, porém dados recentes têm fornecido regras mais acuradas. A UICC (Union Internationale Contra le Cancer) e a AJCC (American Joint Committee on Cancer) definem a margem anal como a pele que se inicia na porção distal da membrana mucosa do canal anal, abaixo da borda anal e o canal anal como se estendendo da porção superior da margem anal proximal até o anel anorretal ${ }^{4,5,6}$. Os tumores da margem anal que não invadem a borda anal são melhor tratados como tumores de pele.

Até a década de 1970, o carcinoma do canal anal era tradicionalmente tratado através de procedimentos cirúrgicos que removiam o esfíncter anal. Em 1974, Nigro et $a l .{ }^{7}$ publicaram os resultados preliminares de três pacientes que foram tratadas com quimioterapia com 5-fluorouracil (5-FU) e mitomicina $\mathrm{C}$ (MMC) e radioterapia concomitantes pré-operatórios, sendo evidenciada resposta completa após o procedimento cirúrgico em dois pacientes e no outro, que recusou cirurgia, não havia recorrência de doença 15 meses após a terapia. A partir de então, numerosos ensaios clínicos têm sido realizados, nos quais os pacientes são tratados com radioterapia e quimioterapia em diversos esquemas, sendo a cirurgia reservada para o resgate, em casos de falha ou para os casos com doença grave e destrutiva do esfíncter anal ${ }^{3,8}$.

O presente artigo tem como objetivo reportar os resultados preliminares do tratamento do carcinoma do canal anal em pacientes atendidos no Hospital do Câncer A. C. Camargo, entre os anos de 1992 e 1998.

\section{MÉTODO}

De janeiro de 1992 a maio de 1998, 24 pacientes foram admitidos com o diagnóstico de neoplasia do canal anal, segundo as definições da UICC e da AJCC para os tumores de canal anal. Foram efetuados história clínica e exame físico completos, seguidos de proctoscopia e biópsia da lesão para análise histológica, em todos os pacientes. Após diagnóstico histológico, os pacientes foram estadiados conforme critérios estabelecidos pela AJCC$\mathrm{UICC}^{5,9}$, que os dividem segundo as categorias T (extensão do tumor primário), N (ausência ou presença e extensão de metástases linfonodais regionais) e M (ausência ou presença de metástases distantes) conforme Tabela 1. Todos foram agrupados em seis classes de estádios (Tabela 2). Realizaram-se, ainda, hemograma, exames bioquímicos e pesquisa de títulos séricos de anticorpos anti-HIV.

O tamanho do tumor primário, conforme avaliação através de toque retal e proctoscopia, variou de 2 a $8 \mathrm{~cm}$, com média de $4 \mathrm{~cm}$. A distribuição do número de pacientes por estádio foi: I - 1, II - 13, III - 9 e IV - 1 .

A distribuição dos pacientes por sexo revelou uma predominância feminina de 3:1 (18 pacientes do sexo feminino e seis do sexo masculino). As idades variaram de 35 a 74 anos, com mediana de 59 anos e a raça mais frequientemente acometida foi a branca, com 19 casos $(79,2 \%)$,
Tabela 1

Estadiamento das neoplasias do canal anal conforme a AJCC e a UICC

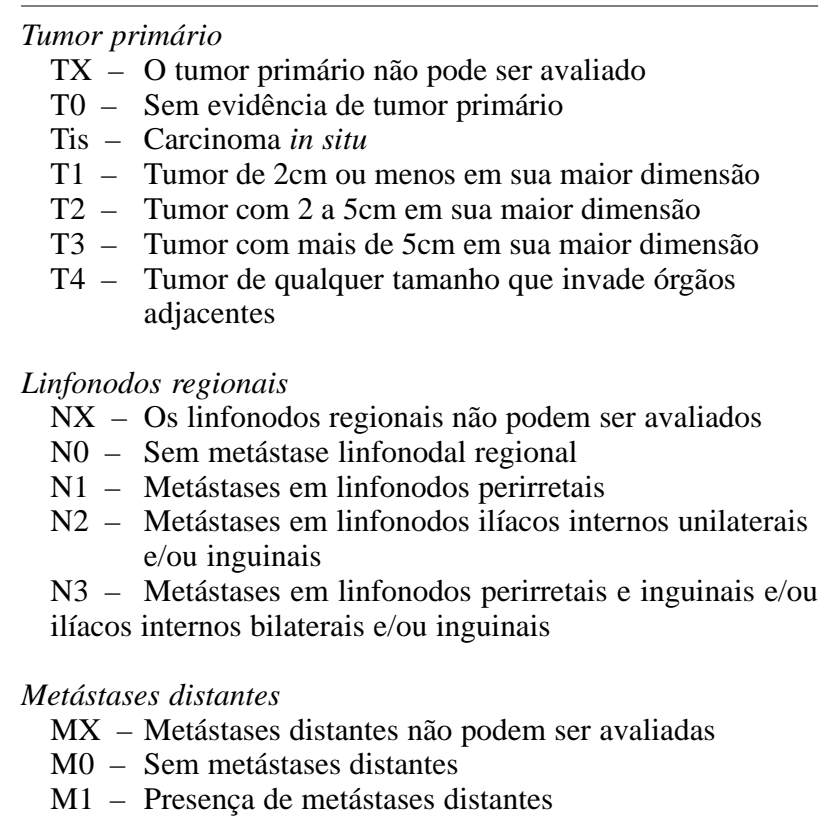

Tabela 2

Grupamento numérico dos estádios clínicos dos tumores do canal anal de acordo com o TNM

\begin{tabular}{c|ccc}
\hline Estádio & \multicolumn{3}{c}{ Grupamento } \\
\hline 0 & Tis & N0 & M0 \\
I & T1 & N0 & M0 \\
II & T2-3 & N0 & M0 \\
IIIA & T1-3 & N1 & M0 \\
& T4 & N0 & M0 \\
IIIB & T4 & N1 & M0 \\
& Qq. T & N2-3 & M0 \\
IV & Qq. T & Qq. N & M1 \\
\hline
\end{tabular}

seguida da negra, com quatro casos $(16,7 \%)$, e da amarela, com um $(4,2 \%)$.

A radioterapia foi realizada no acelerador linear de 4 MV. A primeira fase foi efetivada com dose de 30,6 Gy em 17 frações em campos pélvicos com 3 ou 4 incidências, com limites superior na transição L5-S1, inferior $2 \mathrm{~cm}$ abaixo da extensão caudal do tumor primário e laterais, $2 \mathrm{~cm}$ lateralmente à margem óssea da parede pélvica verdadei$\mathrm{ra}^{10,11}$. Em pacientes com linfonodos inguinais comprometidos clinicamente, esta região foi irradiada através do campo anterior com uso de blocos de transmissão, conforme técnica descrita por Kalend et al. ${ }^{12,13}$. Através desta técnica, coloca-se um bloco de transmissão no campo anterior com o mesmo formato do campo posterior. $\mathrm{O}$ bloco 
possui a finalidade de liberar no meio da espessura do paciente, a metade da dose ( $90 \mathrm{cGy}$ ) que é prescrita a $2 \mathrm{~cm}$ de profundidade (180 cGy) nas regiões inguinais. A metade remanescente da dose ( $90 \mathrm{cGy}$ ) é liberada pelo campo posterior no meio do diâmetro anteroposterior do paciente. As figuras 1 e 2 mostram as radiografias de simulação dos campos pélvicos utilizados na primeira fase do tratamento de incidências anteroposterior e laterais, respectivamente. $\mathrm{Na}$ segunda fase, os campos foram compostos com o limite superior na transição S1-S2, prosseguindo com oito frações de 1,8 Gy, até a dose final de $45 \mathrm{~Gy}$. Na terceira fase do tratamento, foi empregado campo perineal direto na unidade de cobalto com o paciente em posição ginecológica, com a finalidade de complementação de dose no canal anal. Foi prescrita dose de $10 \mathrm{~Gy}$ em cinco frações de $2 \mathrm{~Gy}$ a $3 \mathrm{~cm}$ de profundidade, para obtenção de dose final no leito tumoral de 55 Gy (Figura 3).

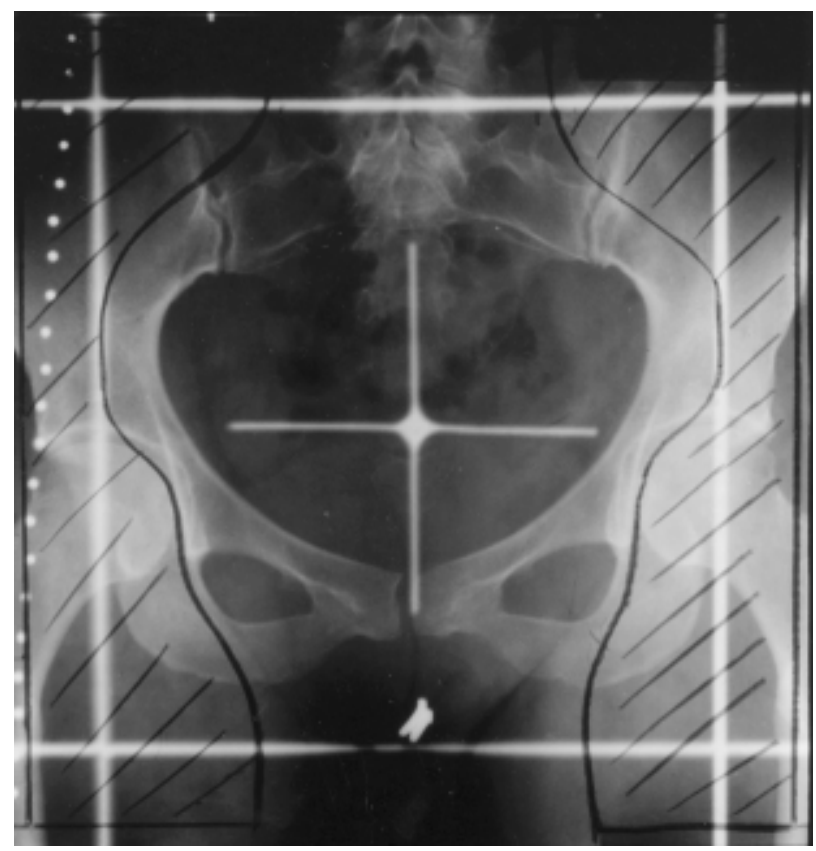

Figura 1 - Raios X de simulação do campo pélvico utilizado na primeira fase do tratamento, quando a drenagem linfática inguinal não é incluída no campo de radiação.

A quimioterapia foi administrada concomitante à radioterapia com 5 -fluorouracil $\left(1.000 \mathrm{mg} / \mathrm{m}^{2}\right.$ de superfície corpórea) em infusão contínua e mitomicina C $\left(10 \mathrm{mg} / \mathrm{m}^{2}\right.$ de superfície corpórea) em bólus, em dois ciclos, durante os cinco primeiros e os cinco últimos dias da radioterapia.

Quinze dias após o término da terapia, todos os pacientes foram submetidos a exame proctológico com biópsia para avaliação da resposta. Naqueles em que a biópsia se apresentou positiva, a mesma foi repetida 45 dias após. Detectando-se resposta completa local, procedia-se ao seguimento, o qual foi realizado através de retornos trimestrais nos dois primeiros anos, semestrais do terceiro ao quinto anos e anuais a partir de então. A anamnese, o exame físico e o exame proctológico foram realizados em todos os retor-

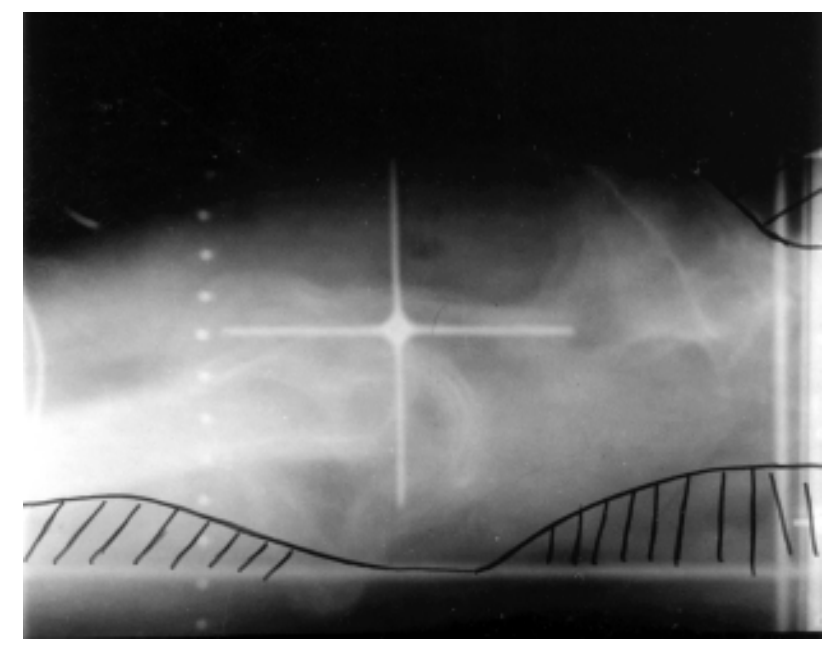

Figura 2 -Raios X de simulação dos campos de incidências laterais

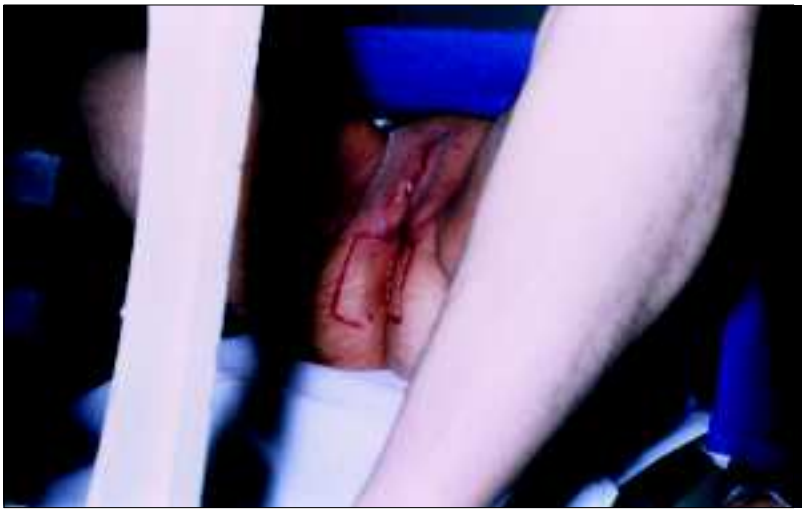

Figura 3 - Posição do paciente para o "boost” perineal.

nos, e radiografia torácica e tomografia computadorizada pélvica, a cada seis meses. Evidenciando-se biópsia positiva, procedia-se à amputação abdominoperineal.

Os efeitos tóxicos do tratamento foram avaliados através de escalas de morbidades agudas e tardias elaboradas pelo RTOG (Radiation Therapy Oncology Group) e pela EORTC (European Organization for Research and Treatment of Cancer $)^{14}$, conforme Tabelas 3 e 4 , respectivamente.

As taxas de sobrevida global foram calculadas de acordo com o método atuarial de Kaplan-Meier ${ }^{15}$.

\section{RESULTADOS}

O seguimento dos pacientes variou de dez a 80 meses, com média de 34 meses. Vinte e três pacientes $(95,8 \%)$ alcançaram resposta completa comprovada por biópsia e em um paciente $(4,2 \%)$, foi evidenciada persistência do tumor. De acordo com a situação atual, $14(58,3 \%)$ pacientes estão vivos e sem doença, cinco $(20,8 \%)$ morreram por câncer, três $(12,0 \%)$ estão vivos com doença e um $(4,2 \%)$ morreu de acidente vascular cerebral sem evidência de câncer.

As falhas do tratamento foram imputadas como recidiva local em cinco pacientes (20,8\%), persistência de doença em um $(4,2 \%)$, metástases a distância em quatro $(16,6 \%)$ 
Tabela 3

Escala do RTOG / EORTC para graduação da morbidade aguda da radioterapia

\begin{tabular}{|c|c|c|c|c|}
\hline Órgão/Tecido & Grau 1 & Grau 2 & Grau 3 & Grau 4 \\
\hline Pele & $\begin{array}{l}\text { Eritema folicular fraco } \\
\text { ou apagado, epilação, } \\
\text { descamação seca, } \\
\text { diminuição da sudorese }\end{array}$ & $\begin{array}{l}\text { Eritema brando ou claro, } \\
\text { descamação úmida em } \\
\text { placas, edema moderado }\end{array}$ & $\begin{array}{l}\text { Descamação úmida } \\
\text { confluente, além das } \\
\text { dobras da pele, edema } \\
\text { em "casca de laranja" }\end{array}$ & $\begin{array}{l}\text { Ulceração, hemorragia, } \\
\text { necrose }\end{array}$ \\
\hline $\begin{array}{l}\text { Gastrointestinal } \\
\text { inferior }\end{array}$ & $\begin{array}{l}\text { Aumento da freqüência } \\
\text { dos hábitos intestinais } \\
\text { não necessitando de } \\
\text { medicação e desconforto } \\
\text { retal não necessitando } \\
\text { de analgésicos }\end{array}$ & $\begin{array}{l}\text { Diarréia necessitando de } \\
\text { medicação e dor retal ou } \\
\text { abdominal necessitando } \\
\text { de analgésicos }\end{array}$ & $\begin{array}{l}\text { Diarréia necessitando de } \\
\text { suporte parenteral, } \\
\text { mucosite grave, perda } \\
\text { de sangue ou distensão } \\
\text { abdominal }\end{array}$ & $\begin{array}{l}\text { Obstrução aguda ou subaguda, } \\
\text { fístula ou perfuração, } \\
\text { sangramento requerendo } \\
\text { transfusão ou dor requerendo } \\
\text { SNG ou cirurgia }\end{array}$ \\
\hline
\end{tabular}

Tabela 4

Escala do RTOG/EORTC para morbidade tardia da radioterapia. Óbito por complicação é considerado grau 5

\begin{tabular}{|c|c|c|c|c|}
\hline Órgão/tecido & Grau 1 & Grau 2 & Grau 3 & Grau 4 \\
\hline Intestinos & $\begin{array}{l}\text { Diarréia branda, leves } \\
\text { cólicas, movimentação } \\
\text { intestinal } 5 x \text { ao dia, leve } \\
\text { descarga retal ou } \\
\text { sangramento }\end{array}$ & $\begin{array}{l}\text { Diarréia moderada e } \\
\text { cólica, movimentação } \\
\text { intestinal 5x ao dia, } \\
\text { excessivo muco retal } \\
\text { ou sangramento } \\
\text { repetitivo }\end{array}$ & $\begin{array}{l}\text { Obstrução ou } \\
\text { sangramento requerendo } \\
\text { cirurgia }\end{array}$ & $\begin{array}{l}\text { Necrose, perfuração ou } \\
\text { fístulas }\end{array}$ \\
\hline Bexiga & $\begin{array}{l}\text { Atrofia epitelial leve, } \\
\text { telangiectasia branda } \\
\text { ou hematúria } \\
\text { microscópica }\end{array}$ & $\begin{array}{l}\text { Freqüência moderada, } \\
\text { telangiectasia } \\
\text { generalizada e hematúria } \\
\text { microscópica repetitiva }\end{array}$ & $\begin{array}{l}\text { Freqüência elevada e } \\
\text { disúria, telangiectasia } \\
\text { generalizada grave, } \\
\text { hematúria freqüente ou } \\
\text { redução na capacidade } \\
\text { vesical (<150cc) }\end{array}$ & $\begin{array}{l}\text { Necrose, bexiga contraída } \\
\text { (capacidade menor do que } \\
100 \mathrm{cc} \text { ) ou cistite hemorrágica } \\
\text { grave }\end{array}$ \\
\hline
\end{tabular}

e segundo tumor primário no pulmão em um (4,2\%). Das falhas locais, três $(12,5 \%)$ eram inicialmente estádio clínico II (T2N0M0), duas (8,3\%) estádio clínico III (um T4N0M0 e um T4N3M0). Não foram observadas recidivas inguinais, nem mesmo nos pacientes com metástases inguinais ao diagnóstico. Para os pacientes que apresentaram recidiva local, o intervalo médio livre de doença foi de 10,4 meses e para os que evoluíram com metástase a distância, de 11,0 meses. A função esfincteriana foi preservada em 18 pacientes (75\%), estando 12 desses $(66,6 \%)$ vivos e sem doença. Quatro pacientes tiveram perda de esfíncter devido à falha local, todas tratadas com cirurgia radical, um devido à persistência de doença e um devido a complicações actínicas.

Complicações agudas ocorreram em 19 (79,2\%) pacientes assim distribuídas: toxicidades cutâneas graus $1 \mathrm{e}$ 2 em $16(66,6 \%)$, gastrointestinais inferiores grau 1 na forma de diarréia em dez $(41,6 \%)$ e de náuseas e vômitos em três $(12,5 \%)$ e toxicidade hematológica grau 1 em sete pacientes $(29,2 \%)$. A interrupção temporária do tratamento, em média de dez dias, foi necessária em 13 pacientes (54,2\%). Complicações tardias ocorreram em nove pacientes $(37,5 \%)$. Um paciente desenvolveu toxicidade genitu- rinária e gastrointestinal inferior grau 1, enquanto os oito restantes apresentaram apenas toxicidade para sistema gastrointestinal inferior com a seguinte distribuição de acordo com a graduação do RTOG: grau I - 5; grau III - 1 e grau IV - 2. A Tabela 5 mostra a situação dos pacientes tratados.

As taxas de sobrevida global atuarial foram de $90 \%$ em dois anos e de 60\% em cinco anos (Figura 4).

\section{DISCUSSÃO}

O carcinoma do canal anal constitui um dos primeiros tumores sólidos em que o tratamento cirúrgico foi eficazmente substituído por técnicas conservadoras, num capítulo curioso da história da oncologia em que uma descoberta aconteceu não motivada por qualquer ensaio clínico randomizado. Com efeito, a descoberta de Norman Nigro não foi primariamente intencional ${ }^{7,16}$ e sim fruto da boa resposta clínica observada em três pacientes tratados com radioterapia e quimioterapia. Três anos após a publicação de seus resultados preliminares, em 1977, Nigro e colaboradores reportaram os resultados do tratamento conservador em dez pacientes, demonstrando respostas completas em seis e parciais em quatro, questionando-se, pela 
Tabela 5

Relação e situação dos pacientes tratados em ordem cronológica

\begin{tabular}{|c|c|c|c|c|c|c|c|c|c|c|}
\hline$N$ & Sexo & Idade & $\begin{array}{l}\text { Estádio } \\
\text { Clínico }\end{array}$ & Resposta & $\begin{array}{l}\text { Preservação } \\
\text { esfincteriana }\end{array}$ & Recaída & Situação & $\begin{array}{c}\text { Grau de } \\
\text { compl. aguda }\end{array}$ & $\begin{array}{l}\text { Interr. } \\
\text { do trat. }\end{array}$ & $\begin{array}{c}\text { Grau de } \\
\text { compl. Crônica }\end{array}$ \\
\hline 1 & $\mathrm{~F}$ & 65 & III & Completa & Não & Local & MOCA & 0 & Não & Retite IV \\
\hline 2 & M & 35 & III & Completa & Sim & Não & SED & 0 & Não & Retite I \\
\hline 3 & $\mathrm{~F}$ & 56 & II & Completa & Sim & Não & SED & III & Sim & Retite + Cistite I \\
\hline 4 & M & 51 & II & Completa & Não & Local & MOCA & III & Não & 0 \\
\hline 5 & $\mathrm{~F}$ & 45 & II & Completa & Sim & Não & SED & IV & Sim & 0 \\
\hline 6 & $\mathrm{~F}$ & 61 & IV & Ausência & Não & Local & MOCA & III & Não & 0 \\
\hline 7 & $\mathrm{~F}$ & 67 & II & Completa & Sim & Não & SED & III & Sim & 0 \\
\hline 8 & $\mathrm{~F}$ & 43 & II & Completa & Sim & Meta & MOCA & 0 & Não & 0 \\
\hline 9 & $\mathrm{~F}$ & 58 & II & Completa & Sim & Não & SED & IV & Sim & Retite I \\
\hline 10 & F & 65 & IV & Completa & Sim & Meta & MOCA & 0 & Não & 0 \\
\hline 11 & $\mathrm{~F}$ & 54 & IV & Completa & Sim & Não & SED & IV & Sim & 0 \\
\hline 12 & $\mathrm{~F}$ & 58 & II & Completa & Sim & Meta & CED & III & Não & Retite I \\
\hline 13 & $\mathrm{~F}$ & 64 & I & Completa & Sim & Não & SED & III & Sim & Retite I \\
\hline 14 & M & 38 & II & Completa & Não & Não & CED & IV & Sim & 0 \\
\hline 15 & $\mathrm{~F}$ & 68 & II & Completa & Sim & Não & SED & III & Sim & Retite I \\
\hline 16 & F & 52 & IV & Completa & Não & Local & SED & IV & Sim & 0 \\
\hline 17 & $\mathrm{~F}$ & 39 & II & Completa & Sim & Não & SED & I & Sim & 0 \\
\hline 18 & $\mathrm{~F}$ & 57 & III & Completa & Sim & Não & SED & III & Não & 0 \\
\hline 19 & M & 62 & II & Completa & Sim & Não & 2.PRIM & III & Não & Retite III \\
\hline 20 & M & 65 & III & Completa & Sim & Não & MSCA & IV & Sim & Retite IV \\
\hline 21 & $\mathrm{~F}$ & 71 & II & Completa & Sim & Não & SED & III & Não & 0 \\
\hline 22 & F & 74 & II & Completa & Sim & Meta & CED & 0 & Não & 0 \\
\hline 23 & M & 60 & II & Completa & Não & Local & SED & IV & Sim & 0 \\
\hline 24 & $\mathrm{~F}$ & 62 & II & Completa & Sim & Não & SED & III & Não & 0 \\
\hline
\end{tabular}

Legendas da situação dos pacientes:

$S E D=$ vivo sem evidência de doença

$C E D=$ vivo com evidência de doença

$M O C A=$ Morte por câncer

$M S C A=$ Morte sem câncer

2.PRIM= Segundo tumor primário

primeira vez na história, a necessidade da ressecção abdominoperineal para estes pacientes ${ }^{17}$. Um programa definitivo de tratamento foi proposto, finalmente, em $1983^{18}$, após uma análise retrospectiva de 104 pacientes tratados com radioterapia e quimioterapia concomitantes. O esquema consistia da administração de $30 \mathrm{~Gy}$ de radioterapia externa, do dia 1 ao dia 21 (D1 a D21) em 15 frações de 2 Gy no tumor primário, na pelve e nos linfonodos inguinais, associada à quimioterapia com 5 -Fluoracil $1.000 \mathrm{mg} / \mathrm{m}^{2} / \mathrm{d}$ em infusão contínua de D1 a D4 e de D28 a D31 e mitomicina C $15 \mathrm{mg} / \mathrm{m}^{2}$ em "bólus" no D1.

Já se encontram bem estabelecidos na literatura os efeitos curativos do tratamento combinado do carcinoma do canal anal com quimioterapia e radioterapia, reservando-se a amputação abdominoperineal para os casos com tumores residuais ou recorrentes após a terapia primária ${ }^{19}$. Esquemas diversos com diferentes drogas já foram testados. Os protocolos variam no que tange às doses e aos intervalos de quimioterapia e de radioterapia ${ }^{19,20}$.

A associação da radioterapia com a quimioterapia foi avaliada em três ensaios randomizados recentes. Um estudo aleatório fase III promovido por instituições européi$\mathrm{as}^{21}$, entre as quais o Instituto Gustave Roussy e a EORTC, analisou 103 pacientes elegíveis e comparou radioterapia exclusiva com radioterapia em regime combinado com 5-fluorouracil e mitomicina C. Os resultados deste estudo revelaram maiores taxas de resposta completa $(80 \%$ x $54 \%$, $\mathrm{p}=0,02)$ e de sobrevida livre de colostomia em cinco anos (aumento de $32 \%, \mathrm{p}=0,02$ ), bem como maior toxicidade aguda, no grupo de tratamento combinado. A sobrevida 


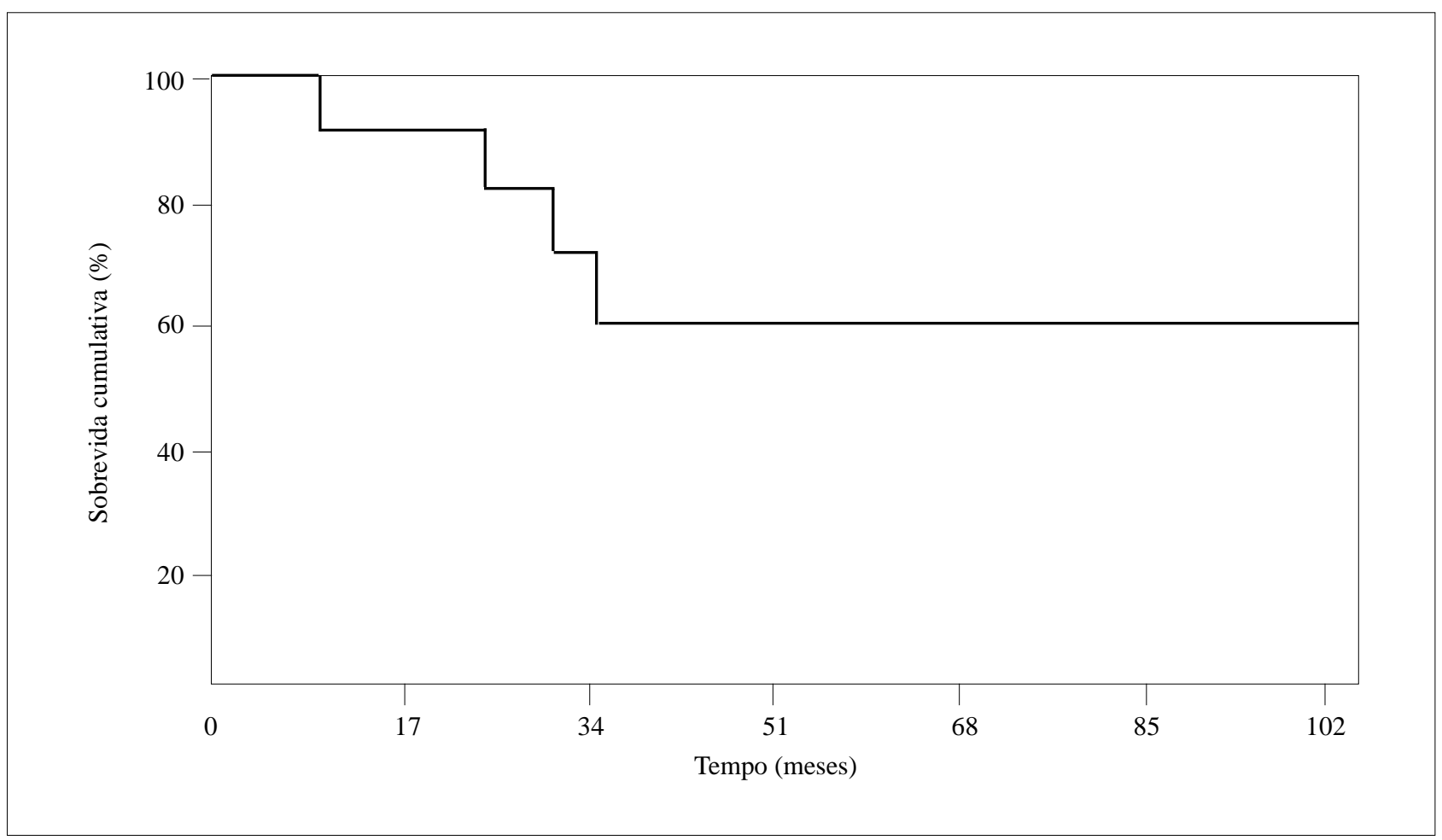

Figura 4 - Gráfico de sobrevida global em função do tempo, em meses, conforme estimativa pelo método de Kaplan-Meier.

global atuarial aos cinco anos foi semelhante nos dois braços. Os principais resultados deste estudo foram o aumento das taxas de controle locorregional e a diminuição do número de colostomias em pacientes com carcinoma do canal anal avançado.

Ensaio clínico também aleatório foi conduzido pela UKCCCR (UK Coordinating Committee on Cancer Research $)^{22}$, com a inclusão de 577 pacientes elegíveis e os braços semelhantes aos do estudo europeu. O controle local de doença foi maior $(59 \%$ x $36 \%)$ e a taxa de recorrência local em três anos menor (39\% x 61\%) para o grupo de tratamento combinado.

$\mathrm{A}$ inclusão da mitomicina $\mathrm{C}$ no regime combinado ocorreu numa base puramente empírica. E uma droga ativa contra outros tumores como gástrico, pancreático e pulmonar. Sua função na terapêutica multimodal do carcinoma do canal anal precisou ser comprovada para que ela fosse categoricamente aceita nos protocolos de tratamento $^{23}$. Seu benefício foi confirmado numa análise retrospectiva realizada no "Princess Margaret Hospital"24, que comparou os resultados de tratamento de pacientes que receberam radioterapia, radioterapia e quimioterapia com 5-fluorouracil e radioterapia e quimioterapia com 5-Fluoracil e mitomicina $\mathrm{C}$. As taxas de controle local foram, respectivamente, $56 \%, 60 \%$ e $86 \%$. Um estudo aleatório do RTOG e ECOG ${ }^{25}$ também analisou se a mitomicina C seria realmente um componente necessário ao esquema de tratamento. Foram avaliados 145 pacientes em um braço recebendo radioterapia e 5-fluoracil e 146 pacientes em outro braço com radioterapia, 5-fluorouracil e mitomicina $\mathrm{C}$, com seguimento médio de três anos. Demonstrou-se um impacto da mitomicina $\mathrm{C}$ na redução da taxa de colostomia, mais significativo entre os tumores mais avançados (T3 e T4). No grupo que recebeu mitomicina $C$, as taxas de sobrevida livre de doença foram maiores (73\% x 51\%), embora as taxas de sobrevida global não tenham diferido estatisticamente. Tais resultados corroboram a necessidade da inclusão da mitomicina $C$ na terapêutica combinada. Ressaltase, neste trabalho, a contra-indicação ao uso da mitomicina C em imunodeprimidos ou HIV positivos, visto que esses pacientes possuem baixa tolerabilidade à neutropenia freqüentemente induzida por tal quimioterápico.

O entendimento da combinação das duas modalidades requer o conhecimento dos mecanismos radiobiológicos e de biologia tumoral. A estratégia que combina radioterapia e quimioterapia concomitantemente minimiza o efeito de resistência cruzada às duas modalidades por parte das células tumorais. Sob o ponto de vista radiobiológico, tal combinação é considerada supra-aditiva, mormente pelo fato que as drogas comumente usadas atuam em pontos distintos do ciclo celular. O principal mecanismo de ação da radiação é de lesão indireta ao DNA já sintetizado na célula, num processo considerado fase-específico, posto que a morte celular se processa principalmente na fase de mitose. O 5-fluorouracil é um antimetabólito que se incorpora diretamente ao RNA como um análogo de uracil, inibindo, desta feita, a síntese do DNA. Tal efeito é observado em células que estão proliferando no ciclo, daí porque se diz ser ele um agente ciclo-específico. A mitomicina $C$ constitui um antibiótico antiblástico com marcada ação contra células hipóxicas ou anaeróbicas, o que torna ani- 


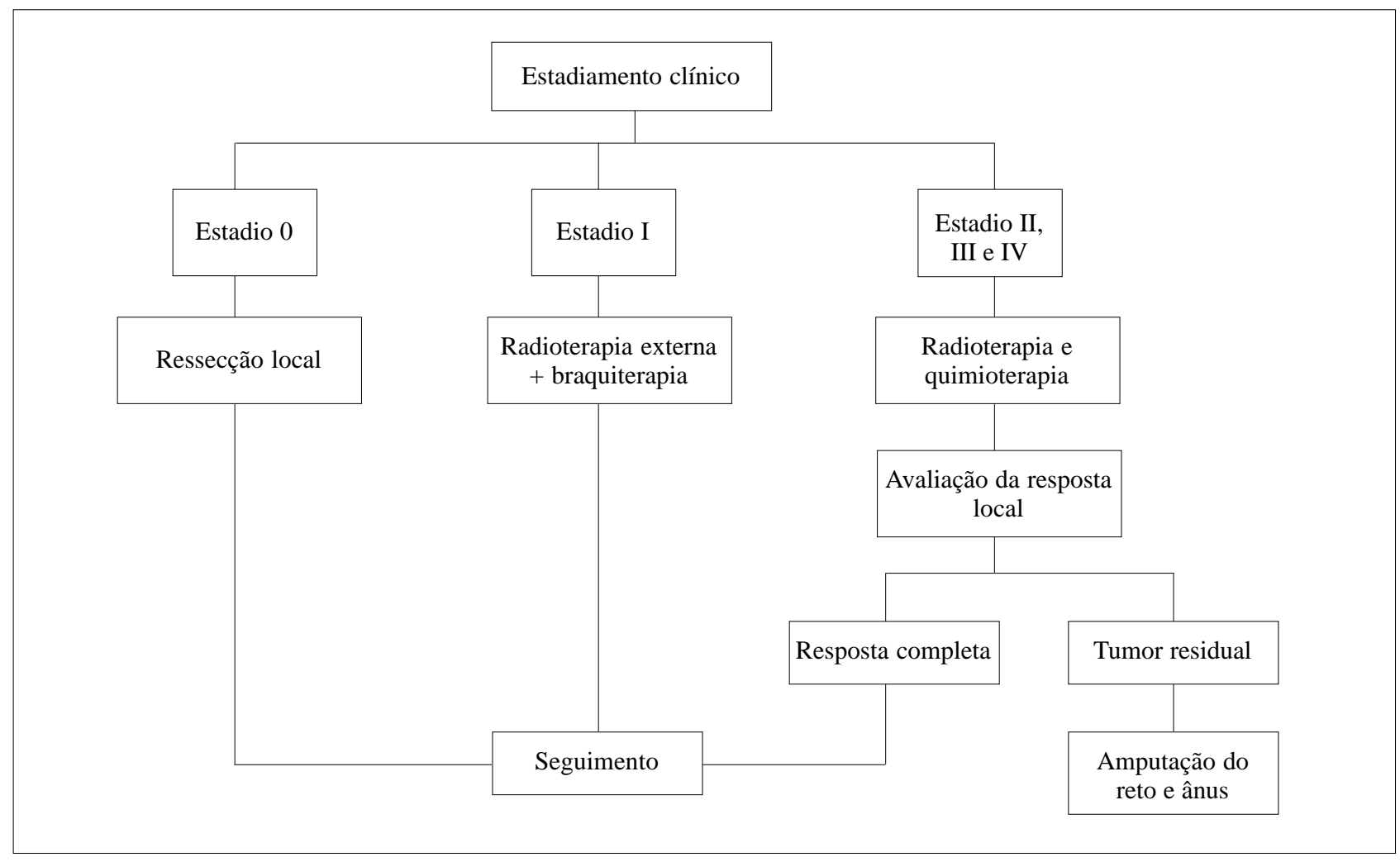

Figura 5 - Organograma de conduta dos tumores do canal anal.

madora, à luz dos conceitos biológicos, a perspectiva de combinação com a radiação. Ela produz monoalquilação do DNA principalmente nas fases G1 tardia e S precoce, fases essas conhecidamente radiorresistentes ${ }^{23,24,26}$.

A associação de 5-fluorouracil e cisplatina em substituição ao clássico esquema com 5-fluorouracil e mitomicina $\mathrm{C}$ foi analisada por Gerard et al. ${ }^{16} \mathrm{em} 1995$ pacientes. As taxas de sobrevidas global e específica em cinco anos foram de $84 \%$ e $90 \%$, respectivamente, com preservação anal em $82 \%$ dos pacientes e toxicidades aguda e tardia aceitáveis. Regimes quimioterápicos baseados em cisplatina deverão, nos próximos anos, ser examinados com maior atenção, visto que tal droga se mostra efetiva em outros carcinomas de células escamosas. Além disso, é necessário o estudo de estratégias que visem à prevenção do aparecimento de metástases distantes, fato que pode acontecer em até $40 \%$ dos pacientes ${ }^{22}$.

Em nossa casuística, houve uma alta taxa de resposta inicial completa ao tratamento combinado $(95,8 \%)$ e um percentual de pacientes com função esfincteriana preservada comparável ao das principais séries da literatura (75\%), com apenas cinco pacientes $(20,8 \%)$ desenvolvendo recidiva local. A toxicidade aguda observada teve alta freqüência $(79,2 \%)$, fato este esperado quando se considera a administração de estratégias potencialmente tóxicas. A morbidade tardia do tratamento consistiu principalmente em complicações de grau leve, passíveis de tratamento clínico. No entanto, um maior número de pacientes e seguimento mais longo nos trarão informações mais precisas sobre esta estratégia terapêutica e manobras para diminuir a toxicidade aguda sem comprometer os resultados de controle local, sobrevida e preservação da função esfincteriana.

A padronização de condutas nos tumores do canal anal foi elaborada de forma multidisciplinar pelos departamentos de radioterapia, cirurgia pélvica e oncologia clínica, e fornece as orientações gerais para o manejo desta doença, conforme organograma da Figura 5.

Alguns pontos ainda merecem debate no que concerne ao manejo desta neoplasia, a saber: a técnica mais adequada de irradiação, a dose final no leito tumoral e o volume de radiação apropriado. Ensaios clínicos de fase III são necessários, ainda, para a completa definição acerca destas questões ${ }^{16}$. Variação de doses de teleterapia entre 45 e 60 Gy tem sido relatada ${ }^{1,16}$. Alguns autores chegam a recomendar dose de radiação de 30,6 Gy para o controle da doença subclínica ${ }^{27}$. Em face dos resultados da maioria dos estudos, consideramos adequada a dose de 45 Gy administrada às áreas de doença subclínica e de 55 Gy ao leito tumoral, quando se utiliza quimioterapia concomitante. Prevemos, como reformulação da nossa padronização de conduta, o emprego da dose de 30,6 Gy para as áreas subclínicas. Esta mudança de dose na pelve provavelmente diminuirá os efeitos colaterais agudos. Para os protocolos que utilizam radioterapia exclusiva, são necessárias doses superiores a $55 \mathrm{~Gy}^{28}$. Um campo a ser investigado é a possível redução da dose de radiação 
para permitir menor tempo de tratamento e diminuição da toxicidade aguda. As interrupções são indesejáveis, posto que, no tratamento de carcinomas escamosos, cada dia de prolongamento da terapêutica exige acréscimo de dose ao plano original da ordem de 0,3 a $0,5 \mathrm{~Gy}^{21}$. A toxicidade aguda representa o fator limitante do tratamento ${ }^{27,29}$. O princípio formulado por alguns autores, o qual reza que, com a associação de drogas quimioterápicas ao tratamento com radioterapia, doses mais baixas de radiação podem ser utilizadas para obtenção das mesmas taxas de controle local e menor índice de complicações, deve ser considerado ${ }^{27,30}$.

Em conclusão, o tratamento standard atual do carcinoma do canal anal, baseado na literatura e confirmado por esta série, é a radioterapia e a quimioterapia concomitantes, reservando a cirurgia de ressecção abdominal para os casos de recidiva local ou ausência de resposta ao tratamento inicial, oferecendo uma chance real ao paciente de cura e preservação da função esfincteriana.

\begin{abstract}
Background: To report our results of anal canal carcinoma treatment with concomitant radiotherapy and chemotherapy. Method: From January 1992 to May 1998, 24 patients with anathomo-pathologic diagnosis of anal canal carcinoma were treated. Age ranged from 35 years old to 74 years old median of 59 years old. Female and male ratio was 3:1. The number of patients per stage was: I - 1, II - 13, III - 9 and IV - 1. Radiotherapy was delivered with doses of $45 \mathrm{~Gy}$ at the whole pelvis with $4 \mathrm{MeV}$ Linear Accelarator followed by boost to the anal canal until 55Gy through direct field at Cobalt unit. Chemotherapy consisted of 5-FU $\left(1000 \mathrm{mg} / \mathrm{m}^{2}\right)$ and Mitomycin $C\left(10 \mathrm{mg} / \mathrm{m}^{2}\right)$ during the first and last five days of radiotherapy. Results: The mean follow-up period was 34 months. Complete response was observed in 23 (95,8\%) patients. Fourteen $(58,3 \%)$ patients are alive with no cancer, $3(12 \%)$ are alive with cancer, 5 (20,8\%) died with cancer and 1 (4,2\%) died with no evidence of cancer. Local recurrences occurred in 5 (20,8\%) and distant metastasis in 4 (16,6\%). All patients with local recurrence were salvaged with abdominal perineal ressection. Sphincter function was preserved in 18(75\%) patients. Acute and chronic complications were observed in $19(79,2 \%)$ and in $9(37,5 \%)$ patients, respectively. Conclusions: The treatment was effective in terms of local control and sphincter preservation, but a with high incidence of acute and late complications. Lower dose of radiation at the whole pelvis should be a reasonable approach to improve acute and late side effects. A larger number of patients and a longer follow-up will give more information about this treatment approach.
\end{abstract}

Key Words: Carcinoma; Anal canal; Radiotherapy; Chemotherapy.

\section{REFERÊNCIAS}

1. Klas JV, Rothenberger DA, Wong WD et al. Malignant tumors of the canal anal: the spectrum of disease, treatment and outcomes. Cancer 1999;85:1683-93.

2. Nadalin W, Aguilar PB, Habr-Gama A et al. Tumores colorretal e canal anal. In: Salvajoli JV, Souhami L, Faria SL, eds. Radioterapia em oncologia. São Paulo: Medsi, 1999: 583-600.

3. Minsky BD. Anal canal cancer. In: Leibel AS, Phillips TL, eds. Textbook of radiation oncology. Philadelphia: WB Saunders, 1998:703-9.

4. Fleming ID, Cooper JS, Henson DE et al. AJCC cancer staging handbook. 5th ed. Philadelphia: Lippincott-Raven, 1998.

5. Hermanek P, Hutter RVP, Sobin LH et al. Atlas TNM: guía ilustrada de la clasificación TNM/pTNM de los tumores malignos. 4th ed. Barcelona: Springer, 1997.

6. Savoca PE, Wong WD. Anal carcinoma: anatomy, staging, and prognostic variables. In: Cohen AM, Winawer SJ, Friedman MA, Gunderson LL, eds. Cancer of the colon, rectum, and anus. New York: McGraw-Hill, 1995: 1013-20.

7. Nigro ND, Vaitkevicius VK, Considine Jr. B. Combined therapy for cancer of the anal canal: a preliminary report. Dis Colon Rectum 1974;17:354-6.
8. Habr-Gama A, Sousa Jr. AHS, Nadalin W et al. Epidermoid carcinoma of the anal canal: results of treatment by combined chemotherapy and radiation therapy. Dis Colon Rectum 1989;32:773-7.

9. Cummings BJ. Anal canal. In: Perez CA, Brady LW, eds. Principles and practice of radiation oncology. Philadelphia: Lippincott-Raven, 1998: 1511-24.

10. Martenson JA, Schild SE, Haddock MG. Cancers of the gastrointestinal tract. In: Khan FM, Potish RA, eds. Treatment planning in radiation oncology. Baltimore: Williams \& Wilkins, 1998:319-42.

11. Martenson Jr. JA, Haddock MG, Gunderson LL. Cancers of the colon, rectum, and anus. In: Levitt SH, Potish RA, Khan FM, Perez CA, eds. Technological basis of radiation therapy. Philadelphia: Lippincott Williams \& Wilkins, 1999:335-47.

12. Kalend AM, Park TL, Wu A et al. Clinical use of a wing field with transmission block for the treatment of the pelvis including the inguinal node. Int J Radiat Oncol Biol Phys 1990;19:153-8.

13. Orton CG, Herskovic AM, Ezzel GA et al. Transmission blocks: clinical and biological rationales. Int J Radiat Oncol Biol Phys 1985;11:2155-8.

14. Cox JF, Stetz J, Pajak TF. Toxicity criteria of the Radiation Therapy Oncology Group (RTOG) and the Euro- 
pean Organization for Research and Treatment of Cancer (EORTC). Int J Radiat Oncol Biol Phys 1995;31:1341-6.

15. Kaplan EL, Meier P. Non parametric estimation from incomplete observation. J Am Stat Assoc 1958;53:457-81.

16. Gerard JP, Ayzac L, Hun D et al. Treatment of anal canal carcinoma with high dose radiation therapy and concomitant fluorouracil-cisplatinum: long-term results in 95 patients. Radiother Oncol 1998;46:249-56.

17. Buroker TR, Nigro N, Bradley G et al. Combined therapy for cancer of the anal canal: a follow-up report. Dis Colon Rectum 1977;20:677-8.

18. Nigro ND. An evaluation of combined therapy for squamous cell cancer of the anal cancer. Dis Colon Rectum 1984;27:763-6.

19. Touboul E, Schlinger M, Buffat L et al. Epidermoid carcinoma of the anal canal: results of curative-intent radiation therapy in a series of 270 patients. Cancer 1994;73:1569-79.

20. Miller EJ, Quan SHQ, Thaler HT. Treatment of squamous cell carcinoma of the anal canal. Cancer 1991;67:2038-41.

21. Bartelink H, Roelofsen F, Eschwege F et al. Concomitant radiotherapy and chemotherapy is superior to radiotherapy alone in the treatment of locally advanced anal cancer: results of a phase III randomized trial of the European Organization for Research and Treatment of Cancer radiotherapy and Gastrointestinal cooperative groups. J Clin Oncol 1997;15:2040-9.

22. UKCCCR Anal Cancer Trial Working Party. Epidermoid anal cancer: results from the UKCCCR randomised trial of radiotherapy alone versus radiotherapy, 5-fluorouracil, and mitomycin. Lancet 1996;348:1049-54.

23. John MJ, Flam MS, Legha SS et al. Chemoradiation: an integrated approach to cancer treatment. Philadelphia: Lea \& Febiger, 1993.

24. Cummings BJ, Keane TJ, O'Sullivan B et al. Epidermoid anal cancer: treatment by radiation alone or by radiation and 5-fluorouracil with and without mitomycin C. Int J Radiat Oncol Biol Phys 1991;21:1115-25.

25. Flam M, John M, Pajak T et al. Role of mitomycin in combination with fluorouracil and radiotherapy, and of salvage chemoradiation in the definitive nonsurgical treatment of epidermoid carcinoma of the anal canal: results of a phase III randomized intergroup study. J Clin Oncol 1996;14:2527-39.
26. Stewart FA, Saunders MI. Combined radiotherapy and chemotherapy: clinical application and evaluation. In: Steel GG, ed. Basic clinical radiobiology. London: Arnold, 1997:195-202.

27. Hughes LL, Rich TA, Delclos L et al. Radiotherapy for anal cancer: experience from 1979-1987. Int J Radiat Oncol Biol Phys 1989;17:1153-60.

28. Martenson JA, Gunderson LL. External radiation therapy without chemotherapy in the management of anal cancer. Cancer 1993;71:1736-40.

29. Allal AS, Obradovic M, Laurencet $F$ et al. Treatment of anal carcinoma in the elderly: feasibility and outcome of radical radiotherapy with or without concomitant chemotherapy. Cancer 1999;85:26-31.

30. Doggett SW, Green JP, Cantril ST. Efficacy of radiation therapy alone for limited squamous cell carcinoma of the anal canal. Int $\mathbf{J}$ Radiat Oncol Biol Phys 1988;15:1069-72.

Endereço para correspondência

Dr. Robson Ferrigno

Hospital do Câncer A.C. Camargo

Departamento de Radioterapia

Rua Prof. Antonio Prudente, 211

01509-010 - São Paulo-SP

E-mail.: rferrigno@uol.com.br 